\title{
Variação Intraespecífica do Lenho de Eugenia uniflora L. em Duas Diferentes Fitofisionomias do Complexo Vegetacional Atlântico
}

\author{
Priscila Alves Marques ${ }^{1}$, Cátia Henriques Callado ${ }^{2}$, \\ Claudia Franca Barros ${ }^{3}$, Cecília Gonçalves Costa ${ }^{3}$ \\ ${ }^{1}$ Programa de Pós-graduação em Ciências Biológicas (Botânica), Universidade Federal do Rio de Janeiro - UFRJ, \\ Rio de Janeiro/RJ, Brasil \\ ${ }^{2}$ Departamento de Biologia Vegetal, Instituto de Biologia Roberto Alcantara Gomes, \\ Universidade do Estado do Rio de Janeiro - UERJ, Rio de Janeiro/RJ, Brasil \\ ${ }^{3}$ Diretoria de Pesquisas, Instituto de Pesquisas Jardim Botânico do Rio de Janeiro, Rio de Janeiro/RJ, Brasil
}

\begin{abstract}
RESUMO
Myrtaceae está entre as principais famílias lenhosas da Floresta Atlântica, destacando-se Eugenia L. como o gênero de maior riqueza de espécies na família. Eugenia uniflora L. apresenta grande representatividade em áreas de restinga, seu ambiente natural, e é amplamente cultivada em outras regiões em função da sua importância econômica. Este estudo investigou a anatomia do lenho de E. uniflora, crescendo em duas fitofisionomias do complexo vegetacional atlântico no Estado do Rio de Janeiro. Dezesseis parâmetros anatômicos foram analisados e revelaram que os elementos celulares dos indivíduos de restinga apresentam maior frequência e são mais curtos e mais largos, e os raios mais baixos e largos do que os dos indivíduos crescendo na Floresta Ombrófila Densa. Os resultados mostraram como as condições ambientais influenciam a estrutura anatômica da madeira e indicam variações intraespecíficas da espécie e de seus mecanismos de adaptação e de sobrevivência no complexo Mata Atlântica.
\end{abstract}

Palavras-chave: Myrtaceae, anatomia ecológica, Mata Atlântica.

\section{Intraspecific Variation in Wood Anatomy of Eugenia uniflora L. in two Plant Communities of the Atlantic Forest Complex}

\begin{abstract}
Eugenia L. is the most species-rich genus of Myrtaceae, one of the most important wood plant families in the Atlantic Rainforest. Eugenia uniflora L. is abundant in the restinga and widely cultivated for economical purposes. This study investigated the wood anatomy of E. uniflora grown in two different plant communities of the Atlantic Rainforest complex in the State of Rio de Janeiro. Sixteen anatomical parameters were analyzed; indicating that in the trees from the Restinga, the fibers are shorter, vessel-elements are more frequent, shorter and larger, and rays are lower and wider than those found in the Dense Ombrophylous Forest. Results showed how environmental conditions influence the anatomical wood structure and reveal intraspecific variations of this species and its mechanisms of adaptation and survival in the Atlantic Rainforest complex.
\end{abstract}

Keywords: Myrtaceae, ecology anatomy, Atlantic rainforest. 


\section{INTRODUÇÃO}

A Floresta Atlântica apresenta elevada biodiversidade e ocorrência de numerosas espécies endêmicas (Myers et al., 2000; Mittermeier et al., 2005). Este bioma encontra-se sob intensa pressão antrópica e está altamente fragmentado, ocupando apenas cerca de $5 \%$ de sua área original (MurraySmith et al., 2009). A falta de um conhecimento mais abrangente sobre o bioma tem sido apontada como a principal dificuldade para a sua conservação (Lima e Guedes-Bruni, 1997; Brandon et al., 2005).

No Estado do Rio de Janeiro, esta floresta apresenta um diversificado conjunto de formações vegetais associadas e inseridas no domínio da Floresta Pluvial Tropical Atlântica, segundo a classificação de Veloso et al. (1991). Predomina a Floresta Ombrófila Densa (das terras baixas, submontana, montana e altomontana) e, em menor proporção, as áreas de formação pioneira de influência marinha (restinga) e fluviomarinha (mata alagadiça, pântano e manguezal), a Floresta Estacional Semidecidual, os campos de altitude e os afloramentos rochosos (Rizzini, 1979; Scarano, 2002). Essa multiplicidade de fitofisionomias confere alta diversidade florística à Floresta Atlântica, o que está relacionado a uma, também, alta diversidade em estratégias de colonização, ajustes biológicos e formas de desenvolvimento das espécies. Segundo Reid et al. (1992), estudar a diversidade significa documentar suas características de composição, distribuição, estrutura e funções; compreender o papel e a função de genes, espécies e ecossistemas; entender os complexos laços que existem entre os ecossistemas modificados e os naturais, e utilizar esse conhecimento para respaldar o desenvolvimento sustentável.

Inúmeros estudos em anatomia vegetal têm sido realizados no sentido de relacionar a estrutura dos vegetais aos diferentes mecanismos de colonização, estabelecimento e desenvolvimento de espécies em seu ambiente natural, e às consequências advindas das modificações desses ambientes. Ênfase especial tem sido atribuída à inter-relação estrutura anatômica e fatores abióticos. Esses fatores exercem forte influência sobre a anatomia dos vegetais, modificando sua estrutura e suas propriedades (tecnológicas, medicinais etc.), podendo intensificar ou mesmo reverter tendências filogenéticas estabelecidas (p. ex.: Baas, 1973; Callado et al., 1997; Mauseth \& Plemons-Rodriguez, 1998; Rôças et al., 1997; Dickison, 2000; Mantuano et al., 2006; Ribeiro \& Barros, 2006; Boeger et al., 2008, 2009). A capacidade de um indivíduo modificar sua morfologia em resposta a um gradiente ambiental constitui uma das mais importantes características dos organismos sésseis, como as plantas. A variabilidade fenotípica é controlada geneticamente e determinada por fatores evolutivos sob limitações ambientais (p.ex. Schlichting, 1986, 2002; Kozlowski et al., 1991). É entendida como um caráter herdável, que sofre a ação da seleção natural (p. ex. Schlichting, 1986; Scheiner, 1993; Via et al., 1995; Valladares et al., 2006) e está relacionada ao ajuste biológico da planta quando em ambientes heterogêneos e sob estresse (p. ex. Duarte, 1998; Bradshaw, 2006; Valadares et al., 2007; Kawamura, 2010).

A diversidade estrutural no xilema secundário está em grande parte condicionada às variações ambientais (Ribeiro \& Barros, 2006), visto que a evolução do lenho é diretamente relacionada à disponibilidade hídrica e ao desenvolvimento de estratégias para maximizar a condutividade hidráulica e diminuir a cavitação e a resistência ao fluxo hidro e aerodinâmico (Ennos, 1999; Sperry, 2003). Os estudos de anatomia ecológica do lenho foram iniciados na década de 1970 (Metcalfe, 1983), destacando-se os trabalhos de Baas (1973) e Carlquist $(1975,1977,1982)$ e, a partir do final do século XX, tiveram grande impulso (p. ex.: Alves \& Angyalossy-Alfonso, 2000, 2002; Noshiro \& Baas, 2000; Woodcock et al., 2000; Carlquist, 2001; YáñezEspinosa et al., 2001, 2004; Liu \& Noshiro, 2003; Lens et al., 2004; Luchi, 2004; Ribeiro \& Barros, 2006; Lima et al., 2009).

Como uma forma de mensurar a ação do ambiente sobre a anatomia da madeira, Carlquist (1977) desenvolveu os índices de vulnerabilidade e mesomorfia. Estes índices partem do princípio de que a alta redundância de elementos de vaso no xilema secundário confere maior segurança fisiológica (Dickison, 2000). Por sua vez, Zimmermann (1983) propõe a utilização do índice de condutividade para aferir a eficiência e a susceptibilidade durante a condução hídrica. A 
utilização desses índices vem sendo questionada pela literatura (p. ex. van den Oever et al., 1981; Carlquist, 2001). Entretanto, sua aplicação em associação com os dados ambientais pode contribuir para a compreensão das estratégias de transporte de água.

Myrtaceae é uma das mais importantes famílias botânicas em comunidades neotropicais (Mori et al., 1983). A família tem sido frequentemente citada em estudos florísticos e/ou fitossociológicos realizados no Estado do Rio de Janeiro, figurando entre as de maior riqueza de espécies (Barroso \& Marques, 1997; Guedes-Bruni, 1998; Kurtz \& Araujo, 2000; Araujo, 2000; Assumpção e Nascimento, 2000; Pereira et al., 2001; Silva-Neto, 2006; Souza et al., 2007; Souza \& Morim, 2008), destacando-se o gênero Eugenia L. como um dos mais representativos na Restinga (Araujo, 2000; Souza \& Morim, 2008) e na Floresta Ombrófila Densa (Barroso \& Peron, 1994; Guedes-Bruni, 1998). Eugenia uniflora L., conhecida popularmente como pitangueira, é uma das espécies mais importantes do gênero, sendo nativa das restingas, mas cultivada amplamente em áreas de Floresta Ombrófila Densa em virtude de sua utilização alimentícia, ornamental e medicinal (Fonseca-Kruel \& Peixoto, 2004). A anatomia da espécie tem sido investigada em estudos de cunho taxonômico (Fontenelle et al., 1994; Soffiatti \& Angyalossy-Alfonso, 1999) e ecológico (Alves et al., 2008).

Este trabalho faz parte de um conjunto de pesquisas que tem por objetivo descrever a estrutura anatômica de espécies da Floresta Atlântica do Estado do Rio de Janeiro e visa a responder as seguintes questões: i) Existem diferenças estruturais entre o lenho de indivíduos de E. uniflora crescendo na Restinga e na Floresta Ombrófila Densa? ii) Em caso positivo, tais diferenças estão de acordo com os parâmetros estabelecidos na literatura para pressões ambientais inerentes a cada um dos ambientes de procedência dos indivíduos analisados?

\section{MATERIAL E MÉTODOS}

O material botânico foi coletado nos seguintes remanescentes de Floresta Atlântica do Estado do Rio de Janeiro: Parque Nacional da Restinga de
Jurubatiba (PNRJ) e Parque Nacional do Itatiaia (PNI).

O PNRJ abrange áreas dos seguintes municípios: Macaé, Carapebus e Quissamã, com uma área total de $148,6 \mathrm{~km}^{2}$. Foram coletadas amostras de indivíduos na formação aberta de Clusia, que se caracteriza por moitas de vegetação densa intercaladas por espaços de areia, em que a cobertura vegetal é esparsa (Araujo et al., 1998). O PNI está localizado na parte mais alta da Serra da Mantiqueira, possui uma área de aproximadamente $300 \mathrm{~km}^{2}$, abrangendo os municípios de Resende e Itatiaia, a sudoeste do Estado do Rio de Janeiro, e os municípios de Bocaina de Minas e Itamonte ao sul do Estado de Minas Gerais (Morim, 2006). Os indivíduos amostrados no PNI estavam a 700 metros de altitude, em região de Floresta Ombrófila Densa montana. Nos dois sítios de estudo, os indivíduos amostrados estavam sob copas de outras árvores, integrando as moitas dominadas por Clusia, no PNRJ, e o sub-bosque de uma área em estado de sucessão secundária, no PNI. A Tabela 1 apresenta uma descrição sucinta das características ambientais de cada sítio de coleta.

Os indivíduos de Eugenia uniflora L. apresentam hábitos diferentes nos dois sítios de coleta. $\mathrm{Na}$ formação aberta de Clusia do PNRJ, são arbustivos, ramificados próximo à base e atingem no máximo um metro de altura, enquanto os indivíduos introduzidos no PNI são arvoretas que atingem até oito metros de altura (Tabela 1). No PNRJ, as amostras foram coletadas dos ramos de maior diâmetro, com utilização de serrote. No PNI, as amostras foram obtidas de espécimes com tronco cilíndrico, reto e sem bifurcação ou defeito aparente, utilizando-se a sonda de Pressler a uma distância de aproximadamente 1,30 m do solo. Das amostras coletadas, confeccionaram-se corpos de prova, posteriormente submetidos a processos de amolecimento (Coradin \& Muñiz, 1991). Em seguida, foram seccionados ao micrótomo de deslize Spencer modelo 860 ou Leica SM 2000R, com espessura média de $16 \mu \mathrm{m}$, orientados nos sentidos transversal e longitudinal tangencial e radial. As secções obtidas foram clarificadas, desidratadas e submetidas à dupla coloração por solução aquosa de Safranina 1\% e de Azul de Astra 1\% (6:4) (Bukatsch, 1972), para confecção de lâminas permanentes (Johansen, 1940). As dimensões das fibras e o comprimento 
Tabela 1. Caracterização geral dos sítios de coleta e dos indivíduos de Eugenia uniflora L. amostrados.

Table 1. Studied sites general characterization and specimens of Eugenia uniflora L. sampled.

\begin{tabular}{lll}
\multicolumn{1}{c}{ Parâmetros } & \multicolumn{1}{c}{ PNRJ* } & \multicolumn{1}{c}{ PNI ${ }^{* *}$} \\
\hline Fitofisionomia & Formação aberta de clusia & Floresta ombrófila densa montana \\
\hline Latitude & $22^{\circ} 00^{\prime}$ e $22^{\circ} 23^{\prime} \mathrm{S}$ & $22^{\circ} 30^{\prime}$ e $22^{\circ} 33^{\prime} \mathrm{S}$ \\
\hline Longitude & $41^{\circ} 15^{\prime}$ e $41^{\circ} 45^{\prime} \mathrm{W}$ & $42^{\circ} 15^{\prime}$ e $42^{\circ} 19^{\prime} \mathrm{W}$ \\
\hline Altitude & Nível do mar & $700 \mathrm{~m} . \mathrm{s} . \mathrm{m}$. \\
\hline Temperatura mínima anual & $20^{\circ} \mathrm{C}$ & $16^{\circ} \mathrm{C}$ \\
\hline Temperatura média anual & $23^{\circ} \mathrm{C}$ & $21^{\circ} \mathrm{C}$ \\
\hline Temperatura máxima anual & $30^{\circ} \mathrm{C}$ & $22^{\circ} \mathrm{C}$ \\
Precipitação anual total & $1.164 \mathrm{~mm}$ & $2.400 \mathrm{~mm}$ \\
\hline Solo & Arenoso & Argiloarenoso \\
\hline Hábito & Arbusto & Arvoreta \\
Condição & Nativa & Introduzida \\
RBw & 8971,8972 & 8979,8980 \\
\hline
\end{tabular}

PNRJ: Parque Nacional da Restinga de Jurubatiba e PNI: Parque Nacional do Itatiaia. RBw: Número de registro na Xiloteca do Instituto de Pesquisas Jardim Botânico do Rio de Janeiro ( ${ }^{\star}$ Araujo et al., 1998; ${ }^{* *}$ Guedes-Bruni, 1998; ${ }^{* *}$ Silva-Neto, 2006).

dos elementos de vaso foram aferidos em material dissociado, pelo método de Jeffrey (Johansen, 1940), e macerado (Dop \& Gautié, 1909).

As descrições, as mensurações e as contagens dos elementos celulares seguiram as recomendações determinadas pela International Association of Wood Anatomists Committee (IAWA, 1989). A porcentagem dos tipos celulares foi calculada segundo Luchi (2004). As imagens foram obtidas por meio do programa Image Pro Plus versão 4.1 para Windows, utilizando-se câmera de vídeo Q Collor R3 acoplada ao microscópio Olympus BX41-BF-I-20 ou câmera de vídeo Coolsnap acoplada ao microscópio Olympus BX50. Os índices de vulnerabilidade, mesomorfia e condutividade foram calculados segundo as fórmulas descritas em Carlquist (1977, 2001). A condutividade hidráulica foi estimada pela equação de Zimmermann (1983), modificada por Fahn et al. (1986).

As características anatômicas - frequência, comprimento, diâmetro e espessura da parede dos elementos vasos; diâmetro das pontoações raiovasculares; comprimento, diâmetro, lume e espessura da parede das fibrotraqueídes; frequência e largura dos raios, e porcentagem dos tipos celulares - foram submetidas a tratamento estatístico utilizando-se o programa Statistica versão 5.0. O teste de Shapiro-Wilks W foi utilizado para testar a normalidade de cada parâmetro analisado (Arango, 2001). Para avaliar a significância da variação entre as populações, aplicou-se o teste $t$ de Student para amostras independentes, quando as variáveis apresentaram distribuição normal (Arango, 2001). O teste de Wilcoxon foi utilizado para as variáveis com distribuição livre (Arango, 2001). Para a visualização das assimetrias das características que se mostraram significativamente diferentes entre os dois sítios de coleta, foram construídos gráficos de Box plot (McGill et al., 1978), no programa Statistica versão 5.0.

\section{RESULTADOS}

O lenho de E. uniflora, nas duas áreas de coleta, apresenta camadas de crescimento distintas, demarcadas por espessamento e achatamento das paredes das fibras no lenho tardio, e maior frequência dos elementos de vaso no lenho inicial (Figuras 1b, 2a). Nos indivíduos do PNI, a zona fibrosa apresenta-se mais larga do que nos indivíduos do PNRJ. A porosidade é difusa; os elementos de vaso são exclusivamente solitários, de contorno circular a oval (Figuras 1a, b, 2a, b), com placas de perfuração simples e apêndices em uma ou em ambas as extremidades (Figura 2f). Raros são os elementos de vaso desprovidos de apêndices. Depósitos de natureza não investigada ocorrem eventualmente nos elementos de vaso. As pontoações raiovasculares são areoladas (Figura 2d). As fibrotraqueídes possuem paredes espessas (Tabela 2), com pontoações nas paredes radiais e tangenciais. $\mathrm{O}$ parênquima axial paratraqueal é escasso (Figura 2b). O parênquima 

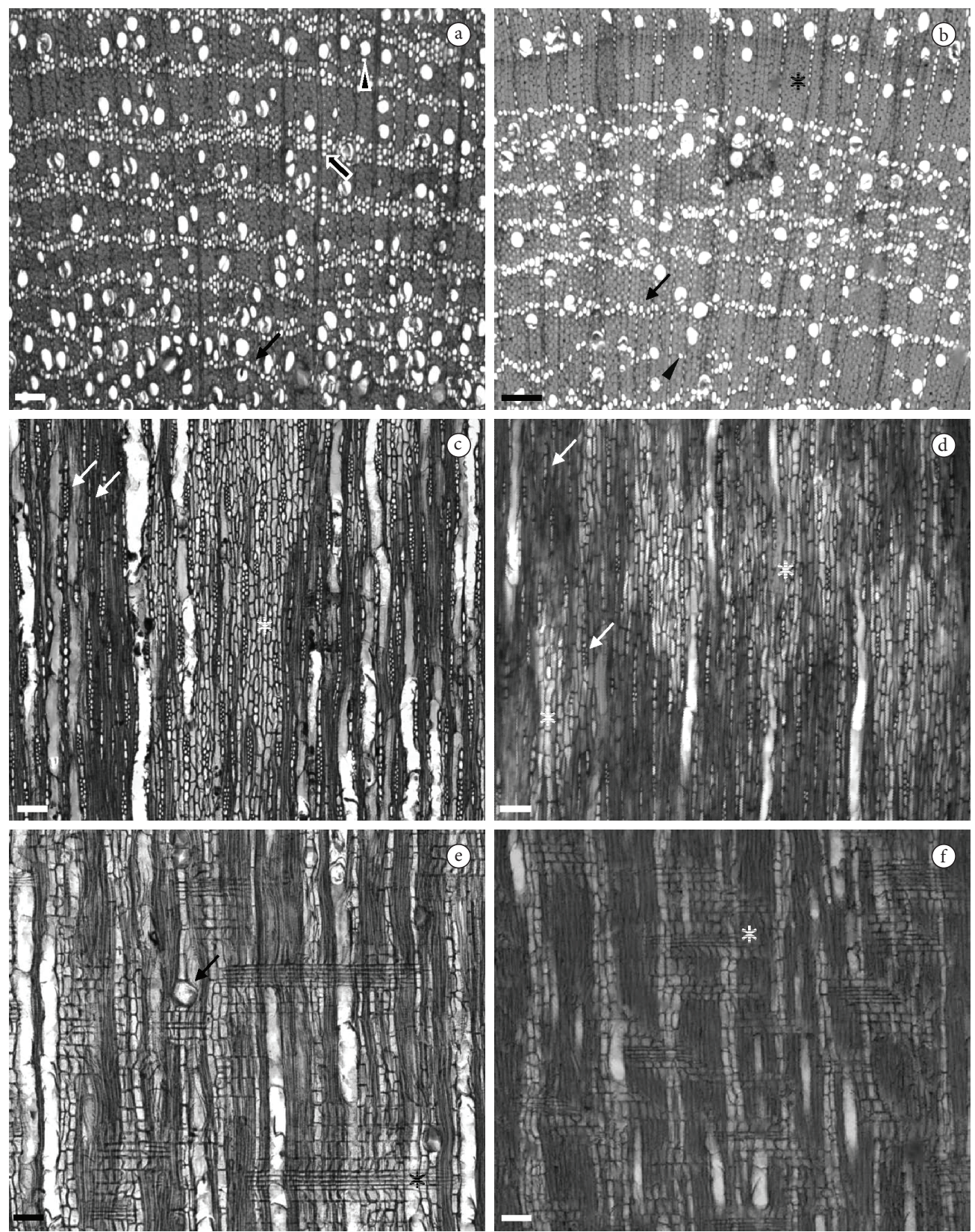

Figura 1. Anatomia do lenho de Eugenia uniflora L. em duas fitofisionomias: Restinga - PNRJ (A, C, E) e Floresta Ombrófila Densa - PNI (B, D, F). a-b) Secção transversal, evidenciando porosidade difusa; elementos de vaso solitários; parênquima apotraqueal difuso e difuso-em-agregados tendendo a formar linhas (seta estreita), parênquima paratraqueal escasso (cabeça de seta) e parênquima apotraqueal em faixa (seta larga). Notar redução do parênquima axial no limite da camada de crescimento (asterisco); c-d) Secção longitudinal tangencial, evidenciando raios unisseriados e localmente bisseriados (seta) e parênquima axial em série (asterisco); e-f) Secção longitudinal radial, evidenciando raios heterogêneos (asterisco) e cristais prismáticos em células amplas do parênquima axial (seta). Barra $=100 \mu \mathrm{m}$.

Figure 1.Wood anatomy of Eugenia uniflora L. in two plant communities: Restinga - PNRJ (A, C, E) and Dense Ombrophylous Forest - PNI (B, D, F). a-b) Transverse section showing diffuse-porous; solitary vessels; diffuse and diffuse-in-aggregates apotracheal axial parenchyma (narrow arrow), scanty paratracheal parenchyma (arrow head) and axial parenchyma bands (large arrow). Note the reduction of the axial parenchyma in the growth rings boundaries (asterisk). c-d) Tangential section showing uniseriate rays and uniseriate rays with biseriate portions (arrow) and strands of axial parenchyma (asterisk); e-f) Radial section showing heterocellular rays (asterisk) and prismatic crystals in enlarged cells of the axial parenchyma (arrow). Bar $=100 \mu \mathrm{m}$. 

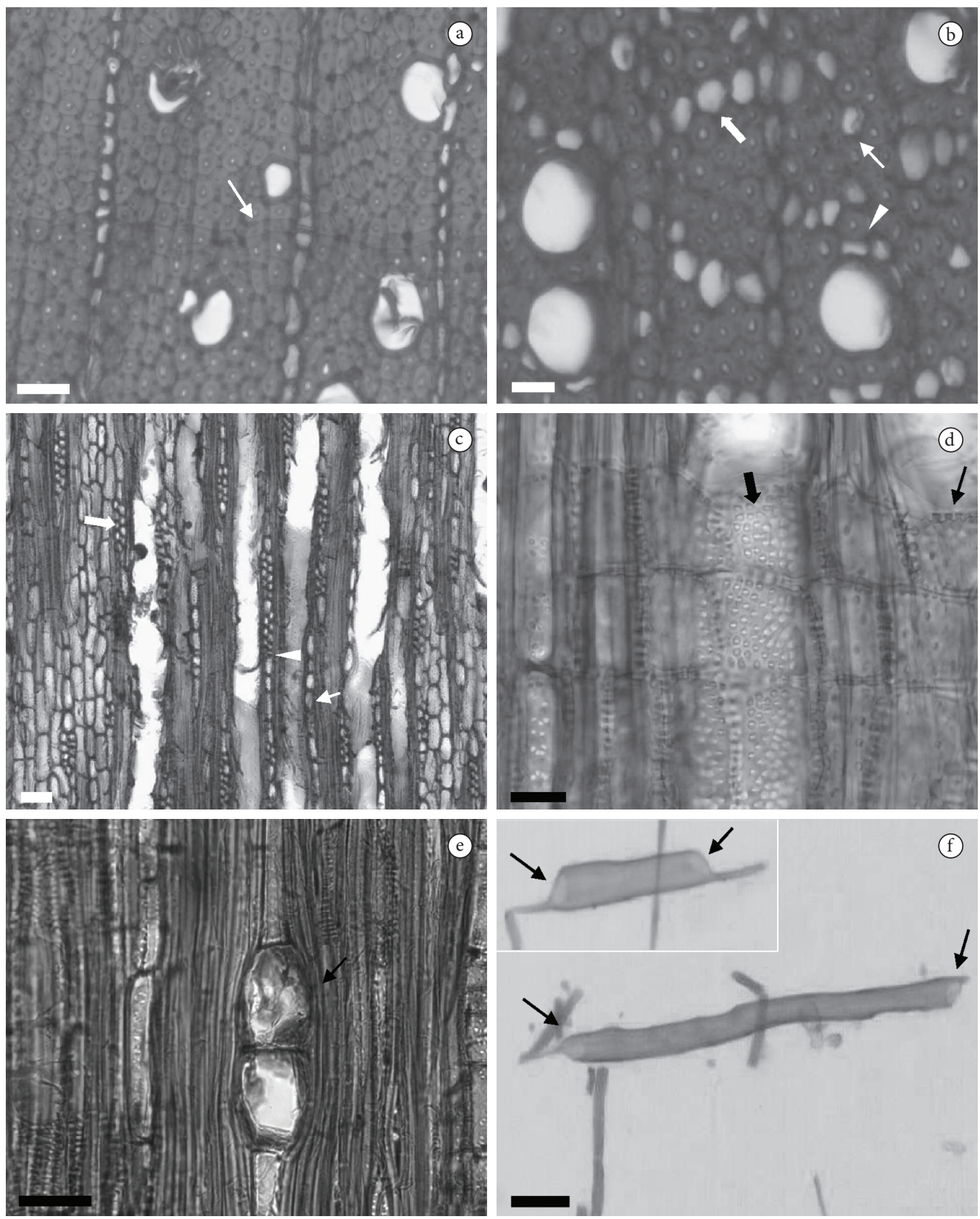

Figura 2. Detalhes da anatomia do lenho de Eugenia uniflora L. a-b) Secção transversal. a) Notar camada de crescimento distinta demarcada pelo achatamento radial das fibras (seta). b) Notar parênquima axial apotraqueal difuso (seta) e difuso-em-agregados (seta larga) e paratraqueal escasso (cabeça de seta); c) Secção longitudinal tangencial, evidenciando raios unisseriados (seta), bisseriados (seta larga) e fusionados (cabeça de seta); d-e) Secção longitudinal radial; d) Notar pontoações raiovasculares areoladas (seta larga) e células disjuntivas do raio (seta); e) Notar cristais prismáticos em células amplas do parênquima axial (seta); f) Elementos de vaso dissociados evidenciando placa de perfuração simples (setas). Barra: $\mathrm{a}=30 \mu \mathrm{m} ; \mathrm{b}, \mathrm{c}$ e e $=50 \mu \mathrm{m} ; \mathrm{d}=20 \mu \mathrm{m} ; \mathrm{f}=100 \mu \mathrm{m}$.

Figure 2. Aspects of the wood anatomy of Eugenia uniflora L. a-b) Transverse section. a) Note the distinct growthrings boundaries, marked by radially flattened latewood fibres (arrow). b) Note diffuse (arrow) and diffuse-in aggregates (large arrow) axial apotracheal parenchyma, and scanty (arrow head) axial paratracheal parenchyma; c) angential section showing unseriate (arrow) and biseriate rays. d-e) Radial longitudinal section; d) Note rounded vessel-rays pits (large arrow) and disjunctive ray parenchyma cells (arrow); e) Note prismatic crystals in wide axial parenchyma cells (arrow); f) Vessel elements showing simple perforation plate (arrow). Bar: $a=30 \mu \mathrm{m} ; \mathrm{b}, \mathrm{c}$ e e $=50$ $\mu \mathrm{m} ; \mathrm{d}=20 \mu \mathrm{m} ; \mathrm{f}=100 \mu \mathrm{m}$. 
Tabela 2. Parâmetros quantitativos (média e desvio padrão) do lenho de Eugenia uniflora L. nas duas fitofisionomias da Floresta Atlântica. As letras indicam diferença significativa entre os resultados comparados em um intervalo de 95\% de confiança; $\mathrm{f}=$ teste $\mathrm{t}$ de student; $\mathrm{z}=$ teste Wilcoxon.

Table 2. Wood quantitative parameters (mean and standard deviation) of Eugenia uniflora L. in two different Atlantic forest physiognomies. The letters indicate significant differences between the results compared in $95 \%$ of confidence intervals; $\mathrm{f}=$ student $\mathrm{t}$ test $; \mathrm{z}=$ Wilcoxon test.

\section{Caracteres anatômicos}

Elementos de vaso

\begin{tabular}{|c|c|c|c|}
\hline Frequência $\left(\mathrm{mm}^{2}\right)$ & $185,2( \pm 47,7)^{\mathrm{a}}$ & $110,7( \pm 10,9)^{b}$ & $\mathrm{z}=3,92$ \\
\hline Comprimento $(\mu \mathrm{m})$ & $450,7( \pm 96,4)^{\mathrm{a}}$ & $519,6( \pm 86,3)^{\mathrm{b}}$ & $\mathrm{f}=1,24$ \\
\hline Diâmetro $(\mu \mathrm{m})$ & $31,7( \pm 5,1)^{\mathrm{a}}$ & $29,7( \pm 4,4)^{b}$ & $\mathrm{f}=1,34$ \\
\hline Espessura da parede $(\mu \mathrm{m})$ & $2,3( \pm 0,4)^{\mathrm{a}}$ & $1,5( \pm 0,4)^{\mathrm{b}}$ & $\mathrm{z}=5,77$ \\
\hline Pontoações raiovasculares $(\mu \mathrm{m})$ & $2,6( \pm 0,2)^{\mathrm{a}}$ & $2,7( \pm 0,3)^{\mathrm{a}}$ & $\mathrm{z}=0,71$ \\
\hline \multicolumn{4}{|l|}{ Fibras } \\
\hline Comprimento $(\mu \mathrm{m})$ & $895,1( \pm 219,8)^{\mathrm{a}}$ & $1122,9( \pm 166,2)^{b}$ & $\mathrm{f}=1,74$ \\
\hline Diâmetro $(\mu \mathrm{m})$ & $16,3( \pm 2)^{\mathrm{a}}$ & $16,5( \pm 1,6)^{\mathrm{a}}$ & $\mathrm{f}=1,64$ \\
\hline Espessura da parede $(\mu \mathrm{m})$ & $6,4( \pm 1)^{\mathrm{a}}$ & $6,5( \pm 0,8)^{\mathrm{a}}$ & $\mathrm{f}=1,63$ \\
\hline Lume $(\mu \mathrm{m})$ & $3,4( \pm 1)^{\mathrm{a}}$ & $3,6( \pm 0,7)^{\mathrm{a}}$ & $\mathrm{f}=1,87$ \\
\hline \multicolumn{4}{|l|}{ Parênquima radial } \\
\hline Frequência (mm) & $14,6( \pm 3,7)^{\mathrm{a}}$ & $14,6( \pm 2,8)^{\mathrm{a}}$ & $\mathrm{f}=1,72$ \\
\hline Altura $(\mu \mathrm{m})$ & $199,9( \pm 6,3)^{\mathrm{a}}$ & $255,9( \pm 89,2)^{b}$ & $\mathrm{f}=1,75$ \\
\hline Largura $(\mu \mathrm{m})$ & $14( \pm 4,4)^{\mathrm{a}}$ & $11,5( \pm 3,3)^{b}$ & $\mathrm{f}=1,89$ \\
\hline \multicolumn{4}{|l|}{ Porcentagem dos tecidos } \\
\hline Elementos de vaso (\%) & $18( \pm 13,2)^{\mathrm{a}}$ & $9,5( \pm 10,5)^{b}$ & $\mathrm{f}=1,92$ \\
\hline Fibras (\%) & $36( \pm 18,4)^{\mathrm{a}}$ & $50,5( \pm 9,4)^{\mathrm{b}}$ & $\mathrm{f}=2,87$ \\
\hline Parênquima radial (\%) & $18( \pm 9,2)^{\mathrm{a}}$ & $17,5( \pm 8,5)^{\mathrm{a}}$ & $\mathrm{f}=0,14$ \\
\hline Parênquima axial (\%) & $28( \pm 14,7)^{\mathrm{a}}$ & $22,5( \pm 9,7)^{\mathrm{a}}$ & $\mathrm{f}=1,22$ \\
\hline
\end{tabular}

axial apotraqueal é difuso e difuso-em-agregados, tendendo a formar linhas e faixas (Figuras 1a, b, 2b). As faixas de parênquima axial são mais visíveis nos indivíduos do PNRJ (Figura 1a). O parênquima axial é seriado (Figura 1c, d) e possui de três a oito células de altura. Cristais prismáticos são observados em células amplas do parênquima axial (Figuras 1, 2e). Os raios são uni e localmente bisseriados e frequentemente se fusionam (Figuras 1c, d, 2c). São compostos por células procumbentes no corpo e por 1 a 4 fileiras de células eretas e/ou quadradas nas margens (Figura 1e, f). Células disjuntivas são frequentemente observadas (Figura 2d).

Nove das dezesseis características quantitativas analisadas no lenho de E. uniflora apresentaram diferenças significativas entre os indivíduos dos diferentes sítios estudados (Tabela 2): frequência, comprimento, diâmetro e espessura da parede dos elementos de vaso; comprimento das fibras; altura e largura de raios, e porcentagem de elementos de vaso e fibras (Figura 3a e i).
Os índices de vulnerabilidade, mesomorfia e condutividade nos dois sítios de coleta encontramse especificados na Tabela 3. Os indivíduos das duas áreas apresentaram índice de vulnerabilidade menor que um e índice de mesomorfia menor que 200. Os valores mais elevados de vulnerabilidade, de mesomorfia e de condutividade foram constatados para os espécimes do PNI.

\section{DISCUSSÃO}

De modo geral, os estudos sobre variação intraespecífica em remanescentes de Floresta Atlântica não evidenciam diferenças significativas em relação aos caracteres qualitativos do lenho de indivíduos crescendo em áreas de diferentes disponibilidades hídricas e altitudes (EsemannQuadros, 2001; Marcati et al., 2001; Ribeiro \& Barros, 2006; Carrera, 2008; Guimarães, 2009). Ao comparar a anatomia do lenho dos indivíduos de E. uniflora crescendo em seu ambiente natural, 

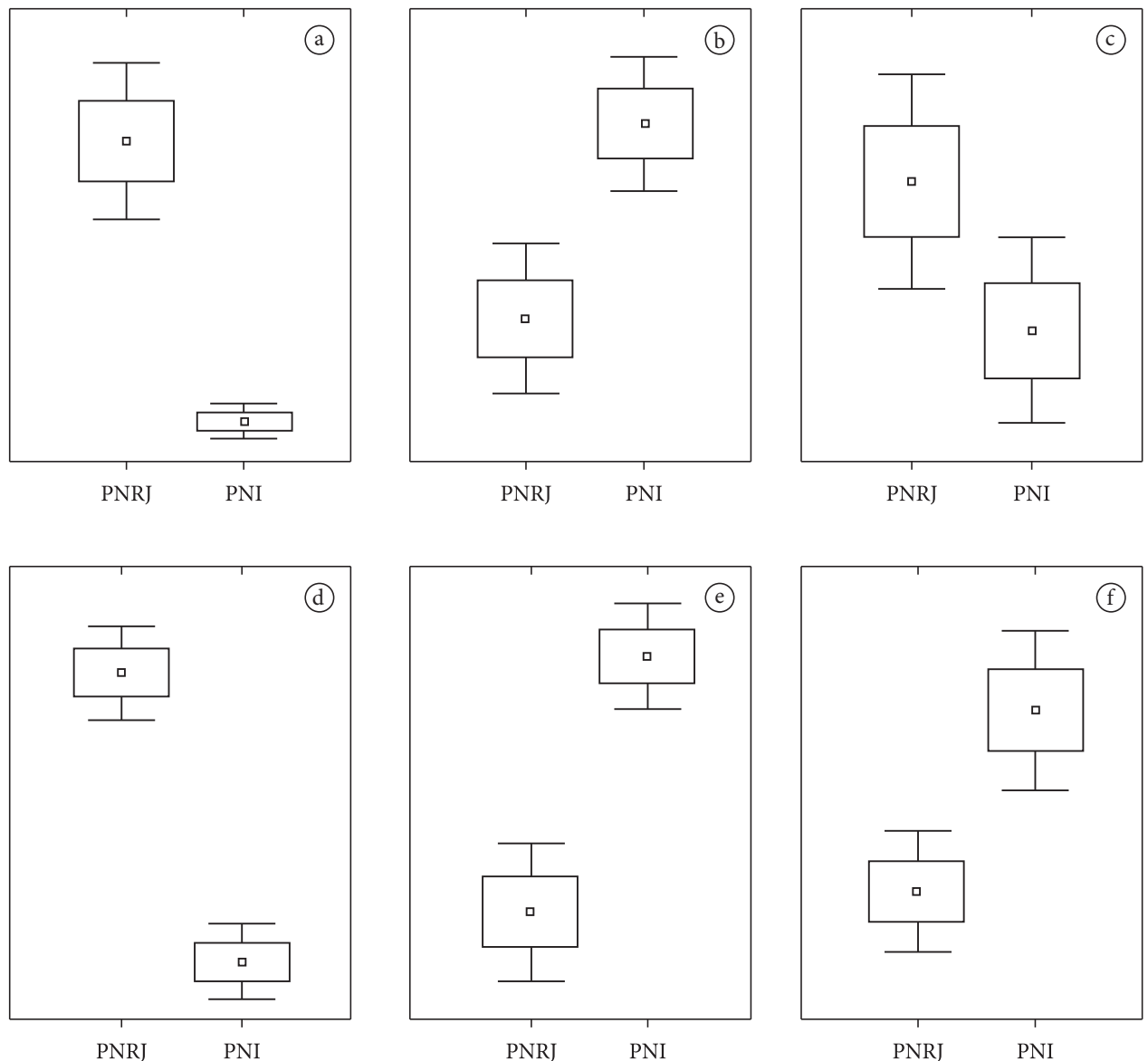

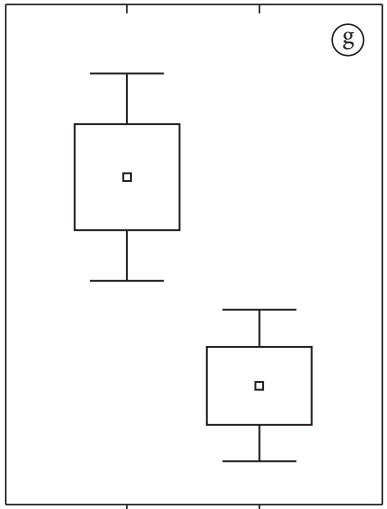

PNRJ

PNI

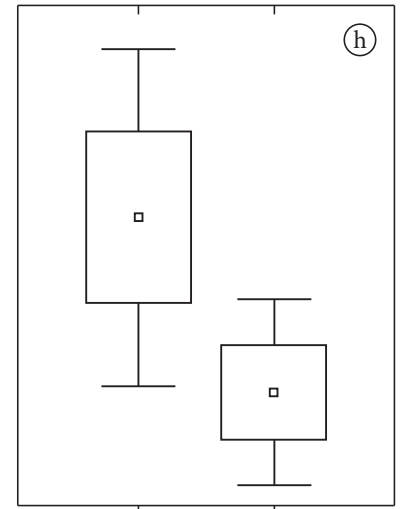

PNRJ

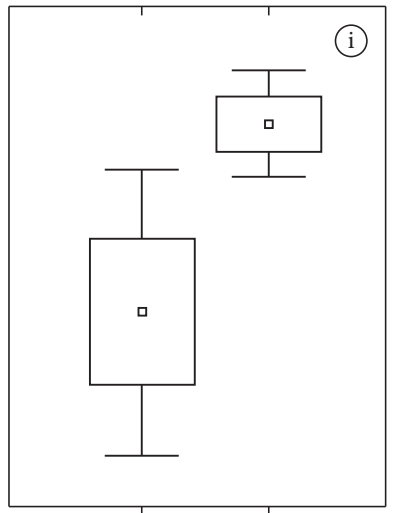

PNRI PNI

Figura 3. Representação em Boxplots dos parâmetros com diferença estatística significativa $(\alpha=95 \%, P<0,05)$ para os caracteres anatômicos de espécimes de Eugenia uniflora L. crescendo em duas fitofisionomias do complexo vegetacional atlântico: Restinga (PNRJ) e Floresta Ombrófila Densa (PNI). Elementos de Vaso (EV); a) Frequência dos elementos de vaso $\left(\mathrm{mm}^{2}\right)$; b) Comprimento dos elementos de vaso $(\mu \mathrm{m})$; c) Diâmetro dos elementos de vaso $(\mu \mathrm{m})$; d) Espessura da parede dos elementos de vaso $(\mu \mathrm{m})$; e) Comprimento das fibras $(\mu \mathrm{m})$; f) Altura dos raios $(\mu \mathrm{m})$; g) Largura dos raios $(\mu \mathrm{m})$; h) Porcentagem dos elementos de vaso; i) Porcentagem das fibras.

Figure 3. Boxplots representation of the statistically significant parameters $(\alpha=95 \%, P<0,05)$ for the anatomical features Eugenia uniflora L. specimens growing in two physiognomies of the Atlantic complex: Restinga (PNRJ) and Dense Ombrophylous Forest (PNI). Vessel elements (EV); a) Vessels frequency $\left(\mathrm{mm}^{2}\right)$; b) Vessels length; ( $\left.\mu \mathrm{m}\right)$; c) Vessels diameter $(\mu \mathrm{m})$; d) Vessels wall thickness $(\mu \mathrm{m})$; e) Fibres length $(\mu \mathrm{m})$; f) Rays height $(\mu \mathrm{m})$; g) Rays width $(\mu \mathrm{m})$; h) Vessels percentage; i) Fibres percentage. 
Tabela 3. Índices de vulnerabilidade, mesomorfia e condutividade hidráulica do xilema secundário de Eugenia uniflora L. nas duas fitofisionomias da Floresta Atlântica.

Table 3. Vulnerability and mesomorphy indexes and hydraulic conductance of the secondary xylem of Eugenia uniflora L. in two physiognomies of the Atlantic forest.

\begin{tabular}{|c|c|c|c|}
\hline Procedência & $\begin{array}{l}\text { Índice de vulnerabilidade } \\
\text { D/F }\end{array}$ & $\begin{array}{c}\text { Índice de mesomorfia } \\
\text { IV } \times C\end{array}$ & $\begin{array}{l}\text { Indice de condutividade } \\
\qquad r^{4} / \mathbf{F}\end{array}$ \\
\hline PNRJ & 0,17 & 77,22 & 341,79 \\
\hline PNI & 0,27 & 139,67 & 442,03 \\
\hline
\end{tabular}

D - Diâmetro médio dos elementos de vaso; F - Frequência média dos elementos de vaso; IV - Índice de vulnerabilidade;

C - Comprimento médio dos elementos de vaso; $r^{4}$ - Média dos raios dos elementos de vaso elevada à quarta potência.

D - mean vessel element diameter; F - mean vessel element frequency; IV - Vulnerability index; C - Mean vessel element length;

$\mathrm{r} 4$ - mean radius of the vessel element raised to the fourth power.

Restinga no PNRJ, e no ambiente onde a espécie foi introduzida, Floresta Ombrófila Densa montana no PNI, também não foram constatadas diferenças estruturais qualitativas conspícuas.

Diversos autores observaram variação quantitativa do lenho relacionada a fatores ambientais, sendo os mais comuns: latitude (Metcalfe, 1983; Wheeler \& Baas, 1991; Dickison, 2000; Noshiro \& Baas, 2000; Liu \& Noshiro, 2003); altitude (Metcalfe, 1983; Wheeler \& Baas, 1991; Dickison, 2000; Noshiro \& Baas, 2000; Liu \& Noshiro, 2003; Lens et al., 2004), e disponibilidade de água (Metcalfe, 1983; Fahn et al., 1986; Villar-Salvador et al., 1997; Dickison, 2000; Luchi, 2004). De acordo com esses autores, quanto maiores a latitude e a altitude, e menor a disponibilidade de água, os elementos de vaso tornam-se mais curtos, estreitos ocorrendo com maior frequência, e as fibras apresentam-se mais curtas e com paredes mais espessas. Os indivíduos de E. uniflora do PNRJ apresentaram elementos de vaso mais curtos, mais largos e em maior densidade, e fibras mais curtas, quando comparados aos indivíduos do PNI. Padrão semelhante foi observado nos indivíduos de Clusia criuva Camb. (Clusiaceae) em Restinga e Floresta Ombrófila Densa montana (Esemann-Quadros, 2001).

A resistência ao fluxo de água e a vulnerabilidade dos vasos dependem da estrutura anatômica do lenho. Elementos de vaso largos podem transportar maior volume de água, porém são mais propensos à embolia (Sperry et al., 1994). Em razão desse fator, as espécies de ambiente com menor disponibilidade de água tendem a apresentar vasos de menor calibre, enquanto o número de vasos tende a aumentar por unidade de área para compensar a eficiência no transporte hídrico (Baas et al., 1983; Metcalfe, 1983; Carlquist, 2001). Fundamentado nas características dos elementos de vaso, Carlquist (1977) estabeleceu índices de mesomorfia e vulnerabilidade. Espécies com índice de mesomorfia superior a 200 são consideradas mesomorfas de acordo com os índices de Carlquist $(1977,2001)$. Na medida em que os indivíduos analisados de E. uniflora apresentaram índice de mesomorfia com valores inferiores a este limite nos dois sítios de coleta, a espécie foi considerada xeromorfa. Todavia, os indivíduos de E. uniflora do PNI apresentam índices menos xeromorfos que os do PNRJ, em função do maior comprimento dos elementos de vaso. Valores elevados do índice de vulnerabilidade indicam maior propensão à cavitação e, consequentemente, à interrupção do fluxo de água (Carlquist, 1977). A despeito de os indivíduos de Restinga apresentarem elementos de vaso mais largos, o índice de vulnerabibilidade encontrado é inferior ao obtido nos indivíduos de Floresta Ombrófila Densa, em função da maior proporção de vasos; portanto, os de Restinga foram considerados mais seguros para o transporte de água. Índices de condutividade mais elevados evidenciam maior eficiência no transporte de água (Carlquist, 2001). Neste estudo, os espécimes de E. uniflora provenientes da Floresta Ombrófila Densa apresentaram valores mais altos de condutividade. Os índices analisados indicaram maior eficiência durante a condução hídrica para os indivíduos do PNI.

No que se refere ao parênquima axial, foi constatada uma tendência ao aumento do tipo em faixas nos indivíduos de Restinga e ao decréscimo de parênquima axial no limite da camada de crescimento 
nos indivíduos do PNI, formando uma distinta zona fibrosa. De modo geral, o aumento na frequência do parênquima axial tem sido relacionado a espécimes de ambientes mais secos (Fahn et al., 1986; Wilkins \& Papassotiriou, 1989; Alves \& Angyalossy-Alfonso, 2002). A análise da porcentagem dos tipos celulares indicou esta diferença, todavia não foi considerada significativa. Marques et al. (2007), comparando espécies de Eugenia de três ambientes distintos da Floresta Atlântica, também no Estado do Rio de Janeiro, observaram que, da mesma forma, a espécie de Restinga apresenta mais parênquima axial. Em E. copacabanensis, o parênquima axial é do tipo em faixa, enquanto as demais espécies de Floresta Ombrófila Densa não apresentam esse caráter. As espécies de Eugenia estudadas por Soffiatti \& Angyalossy-Alfonso (1999) e Barros et al. (2001), oriundas da Floresta Ombrófila Densa, também não apresentam tal característica.

O ambiente também pode influenciar a largura dos raios (Outer \& Veenendaal, 1976; Alves \& Angyalossy-Alfonso, 2002; Luchi, 2004). Alves \& Angyalossy-Alfonso (2002) observaram correlação significativa entre a largura dos raios e a latitude. As autoras também verificaram que, de modo geral, os raios mais largos são observados em ambientes mais úmidos. Entretanto, Luchi (2004) e Lima et al. (2009) registraram a ocorrência de raios mais largos no lenho de espécimes de ambientes com menor disponibilidade hídrica. Esemann-Quadros (2001) não observou diferenças estatisticamente significativas quanto à largura dos raios em C. criuva, mas destacou que a média da largura dos raios é maior em exemplares de restinga quando comparados aos oriundos de mata ciliar. Resultado semelhante e significativo foi obtido para E. uniflora neste trabalho, no qual os raios mais largos foram observados nos indivíduos do PNRJ, cuja disponibilidade de água e nutrientes é considerada menor, e a temperatura e a intensidade de luz, maiores (Araujo \& Henriques, 1984; Araujo et al., 1998; Araujo, 2000; Mantuano et al., 2006; Cavalin \& Mattos, 2007).

Os diferentes hábitos de uma planta (árvores, arbustos, lianas) podem exercer influência significativa sobre os caracteres do lenho (Carlquist, 1982; Olson \& Carlquist, 2001; Lens et al., 2004). Segundo Lens et al. (2004), o hábito arbustivo tem efeito similar aos do estresse hídrico. As árvores, quando comparadas aos arbustos, geralmente apresentam elementos de vaso mais largos, longos e menos frequentes; traqueídes e fibras mais longas, e raios mais baixos e largos (Oslon e Carlquist, 2001; Lens et al., 2004). Em E. uniflora, apenas os resultados encontrados acerca do diâmetro dos elementos de vaso e a altura e a largura dos raios não corroboram a tendência descrita na literatura. Ennos (1999) refere que a eficiência do transporte de água é um elemento crucial para o design de uma planta. Vieira (1990) ressalta que o hábito exerce uma influência indireta sobre a estrutura anatômica, visto que as condições do ambiente determinam o hábito apresentado pela espécie em diferentes sítios e, consequentemente, suas características estruturais.

Em conclusão, os resultados obtidos evidenciam que as condições ambientais influenciam a estrutura anatômica do lenho de E. uniflora. Os indivíduos do PNRJ, que crescem em solo arenoso e estão submetidos a temperaturas mais elevadas e índice pluviométrico mais baixo, apresentam elementos de vaso mais curtos e mais frequentes; fibras mais curtas; raios mais curtos e largos; maior porcentagem de elementos de vaso e de parênquimas axial e radial, e índices de vulnerabilidade, mesomorfia e condutividade menores do que os indivíduos do PNI. Esses resultados evidenciam o investimento em segurança no transporte de água nos indivíduos do PNRJ. Tais resultados podem ser aplicados em estudos que investiguem variações inter e intraespecíficas de espécies lenhosas e seus mecanismos de adaptação e de sobrevivência no complexo Mata Atlântica. Hipótese esta que já está sendo testada para outras espécies de Eugenia, sob as mesmas condições de crescimento.

Em relação à E. uniflora, deve-se destacar, ainda, que a variabilidade anatômica do xilema secundário pode ser considerada vantajosa, uma vez que a interação entre a heterogeneidade ambiental e a variação fenotípica permite que as plantas explorem novos nichos em busca de recursos e ampliem suas possibilidades de distribuição (Villar-Salvador et al., 1997; Cardoso \& Lomônaco, 2003; Sultan, 2003). Deve-se ressaltar que este aspecto é importante principalmente para espécies de interesse econômico. 


\section{AGRADECIMENTOS}

À Dra. Dulce Gilson Mantuano e ao MSc. Arno Fritz Brandes Neves, pelo auxílio na coleta do material botânico; ao Instituto de Pesquisas Jardim Botânico do Rio de Janeiro e à Universidade do Estado do Rio de Janeiro, pelo uso das instalações e dos equipamentos; à CAPES, pela Bolsa de PósGraduação de Priscila Alves Marques; ao CNPq, pela Bolsas de Produtividade de Cátia Henriques Callado, Claudia Franca Barros e Cecília Gonçalves Costa; à FAPERJ e ao CNPq, pelo auxílio financeiro aos projetos aos quais este trabalho está vinculado, $\mathrm{e}$ aos dois revisores anônimos deste trabalho.

\section{STATUS DA SUBMISSÃO}

Recebido: 29/03/2012

Aceito: 30/08/2012

Publicado: 31/12/2012

\section{AUTOR(ES) PARA CORRESPONDÊNCIA}

\section{Cátia Henriques Callado}

Departamento de Biologia Vegetal - DBV,

Instituto de Biologia Roberto

Alcantara Gomes - IBRAG, Universidade do Estado do Rio de Janeiro - UERJ, Rua São Francisco Xavier, 524, PHLC sala 224, Maracanã, CEP 20550-013,

Rio de Janeiro, RJ, Brasil e-mail: ccallado@uerj.br

\section{REFERENCIAS}

Alves ES, Angyalossy-Alfonso V. Ecological trends in the wood anatomy of some brazilian species. 1. Growth rings and vessels. IAWA Journal 2000; 21: 3-30.

Alves ES, Angyalossy-Alfonso V. Ecological trends in the wood anatomy of some Brazilian species. 2. Axial parenchyma, rays and fibres. IAWA Journal 2002; 23: 391-418.

Alves ES, Tresmondi F, Longui EL. Análise estrutural de folhas de Eugenia uniflora L. (Myrtaceae) coletadas em ambiente rural e urbano, SP, Brasil. Acta Botanica Brasilica 2008; 22: 241-248. http://dx.doi.org/10.1590/ S0102-33062008000100023

Arango HG. Bioestatística teórica e computacional. Rio de Janeiro: Guanabara Koogan; 2001.
Araujo DSD, Henriques RBP. Análise florística das restingas do estado do Rio de Janeiro. In: Lacerda LD, Araujo DSD, Cerqueira R, Turcq B, editors. Restingas: origem, estrutura, processos. Niterói: Universidade Federal Fluminense/CEUFF; 1984. p. 159-193.

Araujo DSD. Análise florística e fitogeográfica das restingas do estado do Rio de Janeiro [tese]. Rio de Janeiro: Universidade Federal do Rio de Janeiro; 2000.

Araujo DSD, Scarano FR, Sá CFC, Kurtz BC, Zaluar HLT, Montezuma RCM et al. As Comunidades vegetais do Parque Nacional da Restinga de Jurubatiba, Macaé, RJ. In: Esteves FA, editor. Ecologia das lagoas costeiras do Parque Nacional de Jurubatiba e do município de Macaé. Rio de Janeiro: Universidade Federal do Rio de Janeiro; 1998. p. 39-62.

Assumpção J, Nascimento MT. Estrutura e composição florística de quatro formações vegetais de restinga no complexo lagunar de Grussaí/Iquipari, São João da Barra, RJ, Brasil. Acta Botanica Brasilica 2000; 14: 301-315.

Baas P. The anatomy of Ilex (Aquifoliaceae) and its ecological and phylogenetic significance. Blumea 1973; 21:193-258.

Baas P, Wheeler E, Fahn A. Some ecological trends in vessel characters. IAWA Bulletin 1983; 4:141-159.

Barros CF, Callado CH, Costa CG, Pugialli HRL, Cunha M, Marquete O. Madeiras da Mata Atlântica-Anatomia do lenho de espécies ocorrentes nos remanescentes florestais do estado do Rio de Janeiro - Brasil. Rio de Janeiro: Instituto de Pesquisas Jardim Botânico do Rio de Janeiro; 2001. v. 1.

Barroso GM, Marques MCM. Myrtaceae In: Marques MCM, Vaz ASF, Marquete R, editors. Flórula da Apa Cairuçu, Parati, RJ: espécies vasculares. Rio de Janeiro; 1997. v. 14, p. 314-382. Série e Estudos e Contribuições.

Barroso GM, Perón MV. Myrtaceae. In: Lima MPM, Bruni RRG, editors. Reserva Ecológica de Macaéde Cima, Nova Friburgo, RJ: Aspectos florísticos das espécies vasculares. Rio de Janeiro: Instituto de Pesquisas Jardim Botânico do Rio de Janeiro; 1994. v. 1, p. 261-302.

Boeger MRT, Espíndola-Júnior A, Maccari-Júnior A, Reissmann CB, Alves ACA, Rickli FL. Variação estrutural foliar de espécies medicinais em consórcio com erva-mate, sob diferentes intensidades luminosas. Floresta 2009; 39: 215-225.

Boeger MRT, Gluzezak RM, Pil MW, Goldenberg R Medri M. Variabilidade morfológica foliar de Miconia sellowiana (DC.) Naudin (Melastomataceae) em diferentes fitofisionomias no estado do Paraná. Revista Brasileira de Botânica 2008; 31: 443-452.

Bradshaw AD. Unravelling phenotypic plasticity - why should we bother? New Phytologist 2006; 170: 244-248. http://dx.doi.org/10.1111/j.1469-8137.2006.01761.x 
Brandon K, Fonseca GAB da, Rylands AB, Silva JMC. Conservação brasileira: desafios e oportunidades. Megadiversidade 2005; 1: 7-13.

Bukatsch F. Bemerkungen zur doppelfärbung AstrablauSafranin. Mikrokosmos 1972; 61: 255.

Callado CH, Pugialli HRL, Costa CG, Cunha M, Marquete O, Barros C. Anatomia do lenho de espécies da Mata Atlântica: interpretação ecológica e indicações para aproveitamento. In: Lima HC, Guedes-Bruni RR, editors. Serra de Macaé de Cima: diversidade florística e conservação em Mata Atlântica. Rio de Janeiro: Instituto de Pesquisas Jardim Botânico do Rio de Janeiro; 1997. v. 1 , p. 251-274.

Cardoso GL, Lomônaco C. Variações fenotípicas e potencial plástico de Eugenia calycina Camb. (Myrtaceae) em uma área de transição cerrado-vereda. Revista Brasileira de Botânica 2003; 26: 131-140.

Carlquist S. Ecological strategies of xylem evolution. Berkeley: University of California Press; 1975.

Carlquist S. 1977. Ecological factors in wood evolution: a floristic approach. American Journal of Botany 64: 887-896. http://dx.doi.org/10.2307/2442382

Carlquist S. Wood and bark anatomy of Scalesia (Asteraceae). Aliso 1982; 10: 301-312.

Carlquist SJ. Comparative wood anatomy: systematic, ecological, and evolutionary aspects of dicotyledon wood. 2nd ed. New York: Springer; 2001.

Carrera US. Variação intra-específica na anatomia da folha e do lenho de Tabebuia cassinoides (Lam.) DC. (Bignoniaceae) em ambientes distintos [dissertação]. Rio de Janeiro: Escola Nacional de Botânica Tropical; 2008.

Cavalin PO, Mattos EA. Spatio-temporal variation of photosynthetic pigments in the CAM tree Clusia hilariana Schlechtendal associated with dry spells during the rainy season in southeastern Brazil. Trees 2007; 21: 671-675. http://dx.doi.org/10.1007/ s00468-007-0159-6

Coradin VTR, Muñiz GIB. Normas e procedimentos em Estudos de Anatomia de Madeira: I. Angiospermae. II. Gimnospermae. Laboratório de Produtos Florestais - Série Técnica 1991; 15: 1-19.

Dickison WC. Integrative Plant Anatomy. California: Academy Press; 2000.

Dop P, Gautié A. Manuel de Technique Botanique. Paris: J. Lamane; 1909.

Duarte HM. Variabilidade fenotípica intra $e$ interpopulacional em catacteres morfológicos $e$ anatômicos em populações alopátricas de Quesnelia quesneliana Lindman (Bromeliaceae) sob distintos regimes de luz [dissertação]. Rio de Janeiro: Universidade Federal do Rio de Janeiro; 1998.
Ennos AR. The aerodynamics and hydrodynamics of plants. The Journal of Experimental Biology 1999; 202: 3281-3284.

Esemann-Quadros K. Anatomia do lenho de Clusia criuva Camb. (Clusiaceae): órgãos, hábitos e ambientes [tese]. São Paulo: Universidade de São Paulo; 2001.

Fahn A, Werker E, Baas P. Wood anatomy and identification of trees and shrubs from Israel and adjacent regions. Jerusalém: The Israel Academy of Sciences and Humanities; 1986.

Fonseca-Kruel VS, Peixoto AL. Etnobotânica na Reserva Extrativista Marinha de Arraial do Cabo, RJ, Brasil. Acta Botanica Brasilica 2004; 18: 177-90. http:// dx.doi.org/10.1590/S0102-33062004000100015

Fontenelle GB, Costa CG, Machado RD. Foliar anatomy and micromorphology of eleven species of Eugenia L. (Myrtaceae). Botanical Journal of the Linnaeu Society 1994; 115: 111-133. http://dx.doi. org/10.1111/j.1095-8339.1994.tb00426.x

Guedes-Bruni RR. Composição, estrutura e similaridade florística de dossel em seis unidades fisionômicas de Mata Atlântica no Rio de Janeiro [tese]. São Paulo: Universidade de São Paulo; 1998.

Guimarães RC. Anatomia do lenho de Anadenanthera colubrina (Vell.) Brenan em diferentes fitofisionomias da Floresta Atlântica no estado do Rio de Janeiro: Ilha Grande e Itatiaia [dissertação]. Rio de Janeiro: Escola Nacional de Botânica Tropical; 2009.

International Association of Wood Anatomists - IAWA Commitee. List of microscopic feature of hardwood identification. IAWA Bulletin 1989; 10: 219-332.

Johansen DA. Plant microtechnique. New York: McGraw Hill Book; 1940.

Kawamura K. A conceptual framework for the study of modular responses to local environmental heterogeneity within the plat crown and a review of related concepts. Ecological Research 2010; 25: 733-744. http://dx.doi. org/10.1007/s11284-009-0688-0

Kozlowski TT, Kramer PJ, Pallardy SG. Physiological ecology of woody plants. New York: Academic Press; 1991.

Kurtz BC, Araujo DSD. Composição florística e estrutura do componente arbóreo de um trecho de Mata Atlântica na Estação Ecológica Estadual de Paraíso, Cachoeira de Macacu, Rio de Janeiro, Brasil. Rodriguésia 2000; 51: 69-112.

Lens F, Luteyn JL, Smets E, Jansen S. Ecological trends in the wood anatomy of Vaccinioideae (Ericaceae s.1.). Flora 2004; 199: 309-319. http://dx.doi. org/10.1078/0367-2530-0058

Lima HC, Guedes-Bruni RR. Plantas arbóreas da Reserva Ecológica de Macaé de Cima. In: Lima HC, Guedes-Bruni RR, editors. Serra de Macaé de Cima: 
diversidade florística e conservação em Mata Atlântica. Rio de Janeiro: Jardim Botânico do Rio de Janeiro; 1997. p. 53-63.

Lima RS, Oliveira PL, Rodrigues LR. Anatomia do lenho de Enterolobium contortisiliquum (Vell.) Morong (Leguminosae-Mimosoideae) ocorrente em dois ambientes. Revista Brasileira de Botânica 2009; 32: 361-374.

Liu J, Noshiro S. Lack of latitudinal trends in wood anatomy of Dodonaea viscosa (Sapindaceae), a species with a worldwide distribution. American Journal of Botany 2003; 90: 532-539. http://dx.doi.org/10.3732/ ajb.90.4.532

Luchi AE. Anatomia do lenho de Croton urucurana Baill. (Euphorbiaceae) de solos com diferentes níveis de umidade. Revista Brasileira de Botânica 2004; 27: 271-280.

Mantuano DG, Barros CF, Scarano FR. Leaf anatomy variation within and between three "restinga" populations of Erythroxylum ovalifolium Peyr. (Erythroxylaceae) in southeast Brazil. Revista Brasileira de Botânica 2006; 29: 209-215.

MarcatiCR, Angyalossy-Alfonso V, BenetatiL. Anatomia comparada do lenho de Copaifera langsdorffii Desf. (Leguminosae-Caesalpinoideae) de floresta e cerradão. Revista Brasileira de Botânica 2001; 24: 311-320.

Marques PA, Araujo GUC, Barros CF, Callado $\mathrm{CH}$. Anatomia do lenho de três espécies de Eugenia L. (Myrtaceae) de mata e restinga. Revista Brasileira de Biociências 2007; 5: 801-803.

Mauseth JD, Plemons-Rodriguez BJ. Evolution of extreme xeromorphic characters in wood: a study of nine evolutionary lines in Cactaceae. American Journal of Botany 1998; 85: 209-218. http://dx.doi. org/10.2307/2446308

McGill R, Tukey JW, Larsen WA. Variations of box plots. The American Statistician 1978; 32:12-16.

Metcalfe CR. Ecological anatomy and morphology. General survey. In: Metcalfe CR, Chalk L, editors. Anatomy of the dicotyledons. 2nd ed. Oxford: Clarendon Press; 1983. v. 2.

Mittermeier RA, Gil PR, Hoffman M, Pilgrim J, Brooks T, Mittermeier CG et al. Hotspots revisited: earth's biologically richest and most endangered terrestrial ecoregions. Chicago: University of Chicago Press; 2005.

Mori SA, Boom BM, Carvalino AM, Santos TS Ecological importance of Myrtaceae in a eastern Brazilian wet forest. Biotropica 1983; 15: 68-70. http:// dx.doi.org/10.2307/2388002

Morim MP. Leguminosae arbustivas e arbóreas da Floresta Atlântica do Parque Nacional do Itatiaia, sudeste do Brasil: padrões de distribuição. Rodriguésia 2006; 57: 27-45.
Murray-Smith C, Brummitt NA, Oliveira-Filho AT, Bachman S, Moat J, Nic Lughada EM et al. Plant Diversity Hotspots in the Atlantic Coastal Forests of Brazil. Conservation Biology 2009; 23: 151-163. http:// dx.doi.org/10.1111/j.1523-1739.2008.01075.x

Myers N, Mittermeier RA, Mittermeier CG, Da Fonseca GAB, Kent J. Biodiversity hotspots for conservation priotities. Nature 2000; 403: 853-858. http://dx.doi. org/10.1038/35002501

Noshiro S, Baas P. Latitudinal trends in wood anatomy within species and genera: case study in Cornus S.L. (Cornaceae). American Journal of Botany 2000; 87: 1495-1506. http://dx.doi. org/10.2307/2656876

Olson ME, Carlquist S. Stem and root anatomical correlations with life form diversity, ecology, and systematics in Moringa (Moringaceae). Botanical Journal of the Linnaeu Society 2001; 135: 315-348. http:// dx.doi.org/10.1111/j.1095-8339.2001.tb00786.x

Outer RW, Veenendaal WLH. Variation in wood anatomy of species with a distribution covering both rain forest and savanna areas of the Ivory Coast, WestAfrica, Leiden. In: Baas P, Bolton AJ, Catling DM, editors. Wood structure in biological and technological research. Leiden: Leiden University Press;1976. p. 182-195. Leiden Botanical Series, n. 3.

Pereira MCA, Araujo DSD, Pereira OJ. Estrutura de uma comunidade arbustiva da restinga de Barra de Maricá - RJ. Revista Brasileira de Botânica 2001; 24: 273-281.

Reid W, Barber C, Miller K. A estratégia global para biodiversidade: guia para aqueles que tomam decisão. Curitiba: Fundação o Boticário de Proteção à Natureza; 1992.

Ribeiro MLRC, Barros CF. Variação intraspecífica do lenho de Pseudopiptadenia contorta (DC.) G.P. Lewis \& M.P. Lima (Leguminosae - Mimosoideae) de populações ocorrentes em dois remanescentes de Floresta Atlântica. Acta Botanica Brasilica 2006; 20: 839-844. http://dx.doi. org/10.1590/S0102-33062006000400009

Rizzini CT. Tratado de fitogeografia do Brasil. São Paulo: EdUSP; 1979.

Rôças G, Barros CF, Scarano FR. Leaf anatomy plasticity of Alchornea triplinervia (Euphorbiaceae) under distinct light regimes in a Brazilian montane Atlantic rain forest. Trees 1997; 11: 469-473. http://dx.doi.org/10.1007/ PL00009688

Scarano FR. Structure, Function and Floristic Relationships of Plant Communities in Stressful Habitats Marginal to the Brazilian Atlantic Rainforest. Annals of Botany 2002; 90: 517-524. http://dx.doi. org/10.1093/aob/mcf189 
Scheiner SM. Genetics and evolution of phenotypic plasticity. Annual Review of Ecology, Evolution, and Systematics 1993; 24: 35-68. http://dx.doi.org/10.1146/ annurev.es.24.110193.000343

Schlichting CD. Phenotypic plasticity in plants. Plant Species Biology 2002; 17: 85-88. http://dx.doi. org/10.1046/j.1442-1984.2002.00083.x

Schlichting CD. The evolution of phenotypic plasticity in plants. Annual Review of Ecology, Evolution, and Systematics 1986; 17: 667-694. http://dx.doi. org/10.1146/annurev.es.17.110186.003315

Silva-Neto SJ. A família Rubiaceae Juss. no Parque Nacional do Itatiaia, Itatiaia, $R J$ [tese]. Rio de Janeiro: Universidade Federal do Rio de Janeiro; 2006.

Soffiatti P, Angyalossy-Alfonso V. Estudo anatômico comparativo do lenho e da casca de duas espécies de Eugenia L. (Myrtaceae). Revista Brasileira de Botânica 1999; 22: 175-184.

Souza MC, Morim MP. Subtribos Eugeniinae O. Berg e Myrtinae O. Berg (Myrtaceae) na Restinga da Marambaia, RJ, Brasil. Acta Botanica Brasilica 2008; 22: 652-683. http://dx.doi.org/10.1590/ S0102-33062008000300006

Souza MC, Morim MP, Conde MMS, Menezes LFT. Subtribo Myrciinae Berg (Myrtaceae) na Restinga de Marambaia, RJ, Brasil. Acta Botanica Brasilica 2007; 21: 49-63. http://dx.doi.org/10.1590/ S0102-33062007000100006

Sperry JS, Nichols KL, Sullivan JE, Eastlack SE. Xylem embolism in ring-porous, diffuse-porous, and coniferous trees of northern Utah and interior Alaska. Ecology 1994; 75: 1736-1752. http://dx.doi. org/10.2307/1939633

Sperry JS. Evolution of water transport and xylem structure. International Journal of Plant Science 2003; 164: 115-127. http://dx.doi. org/10.1086/368398

Sultan SE. Phenothypic plasticity in plants: a case study in ecological development. Evolution and Development 2003; 5: 25-33. http://dx.doi.org/10.1046/ j.1525-142X.2003.03005.x

Valadares F, Gianoli E, Gomez JM. Ecological limits to plant phenotypic plasticity. New Phytologist 2007; 176: 749-763. http://dx.doi. org/10.1111/j.1469-8137.2007.02275.x
Van den Oever L, Baas P, Zandee M. Comparative wood anatomy of Symplocos and latitude ana altitude of provenance. IAWA Bulletin 1981; 2: 3-24.

Valladares F, Sanchez D, Zavala MA. Quantitative estimation of phenotypic plasticity: bridging the gap between the evolutionary concept and its ecological applications. Journal of Ecology 2006; 94: 1103-1116. http://dx.doi.org/10.1111/j.1365-2745.2006.01176.x

Veloso HP, Rangel-Filho ALR, Lima JCA. Classificação da vegetação brasileira adaptada a um sistema universal. Rio de Janeiro: Instituto Brasileiro de Geografia e Estatística; 1991.

Via S, Gomulkiewicz R, De Jong G, Scheiner SM, Schlicting CD, Van Tienderen PH. Adaptive phenotypic plasticity: consensus and controversy. Trends in Ecology \& Evolution 1995; 10: 212-217. http://dx.doi. org/10.1016/S0169-5347(00)89061-8

Vieira RC. Contribuição ao conhecimento da anatomia ecológica de Bauhinia radiata Vell [dissertação]. Rio de Janeiro: Universidade Federal do Rio de Janeiro; 1990.

Villar-Salvador P, Castro-Díez P, Pérez-Rontomé C, Montserrat-Martí G. Stem xylem features in three Quercus (Fagaceae) species along a climatic gradient in NE Spain. Trees 1997; 12: 90-96.

Wheeler EA, Baas P. A survey of the fossil record for dicotyledonous wood and its significance for evolutionary and ecological wood anatomy. IAWA Bulletin 1991; 12: 275-332.

Wilkins AP, Papassotiriou S. Wood anatomical variation of Acacia melanoxylon in relation to latitude. IAWA Bulletin 1989; 10: 201-207.

Woodcock DW, Dos Santos G, Reynel C. Wood characteristics of Amazon forest types. IAWA Bulletin 2000; 21: 277-292.

Yáñez-Espinosa L, Terrazas T, López-Mata L. Effects of flooding on wood and bark anatomy of four species in a mangrove forest community. Trees 2001; 15: 91-97. http://dx.doi.org/10.1007/s004680000081

Yáñez-Espinosa L, Terrazas T, López-Mata L, ValdezHernández JI. Wood variation in Laguncularia racemosa and its effect on fibre quality. Wood Science and Technology 2004; 38: 217-226. http://dx.doi. org/10.1007/s00226-004-0228-6

Zimmermann MH. Xylem structure and the ascent of sap. Berlin: Springer-Verlag, 1983. 